\title{
Systematic review of community participation interventions to improve maternal health outcomes in rural South Asia
}

Binod Bindu Sharma ${ }^{1,2^{*}}$, Lisa Jones ${ }^{3,4}$, Deborah Joanne Loxton ${ }^{5,6}$, Debbie Booth ${ }^{2}$ and Roger Smith ${ }^{7,8,9}$

\begin{abstract}
Background: This is a systematic review on the effectiveness of community interventions in improving maternal health care outcomes in South Asia.

Methods: We searched electronic databases to June 2017. Randomised or cluster randomised studies in communities within rural/remote areas of Nepal, Bangladesh, India and Pakistan were included. Data were analysed as risk ratios (RR) or odds ratios (OR), and effects were adjusted for clustering. Meta-analyses were performed using random-effects and evidence quality was assessed.
\end{abstract}

Results: Eleven randomised trials were included from 5440 citations. Meta-analysis of all community interventions combined compared with control showed a small improvement in the number of women attending at least one antenatal care visit (RR 1.19, 95\% Cl 1.06 to 1.33). Two community mobilisation sub groups: home care using both male and female mobilisers, and education by community mobilisers, improved the number of women attending at least one antenatal visit. There was no difference in the number of women attending at least one antenatal visit for any other subgroup. There was no difference in the number of women attending 3 or more antenatal visits for all community interventions combined, or any community subgroup. Likewise, there was no difference in attendance at birth between all community interventions combined and control. Health care facility births were modestly increased in women's education groups (adjusted RR (1.15,95\% Cl 1.11 to 1.20; 2 studies)). Risk of maternal deaths after 2 years (RR 0.63, 95\% Cl 0.24 to $1.64 ; 5$ studies), and 3 years (RR 1.11, 95\% Cl 0.52 to 2.36; 2 studies), were no different between women's education groups and control. Community level mobilisation rather than health care messages at district level improved the numbers of women giving birth at health care facilities (RR1.09 (95\%Cl 1.06 to 1.13; 1 study)). Maternal health care knowledge scores improved in two community-based interventions, one involving education of male community members.

Conclusion: Women's education interventions may improve the number of women seeking birth at a health care facility, but the evidence is of low quality. No impact on maternal mortality was observed Future research should explore the effectiveness of including male mobilisers.

Trial registration: This systematic review is registered with PROSPERO CRD42016033201.

Keywords: Community, Networks, Rural, Pregnancy, Antenatal care, Delivery, Pregnancy complications, Maternal death

\footnotetext{
* Correspondence: BinodBindu.Sharma@uon.edu.au

${ }^{1}$ Mothers and Babies Research Centre, Hunter Medical Research Institute,

Lookout Rd, New Lambton Heights, NSW 2305, Australia

${ }^{2}$ The University of Newcastle, Callaghan, Australia

Full list of author information is available at the end of the article
}

(c) The Author(s). 2018 Open Access This article is distributed under the terms of the Creative Commons Attribution 4.0 International License (http://creativecommons.org/licenses/by/4.0/), which permits unrestricted use, distribution, and reproduction in any medium, provided you give appropriate credit to the original author(s) and the source, provide a link to the Creative Commons license, and indicate if changes were made. The Creative Commons Public Domain Dedication waiver (http://creativecommons.org/publicdomain/zero/1.0/) applies to the data made available in this article, unless otherwise stated. 


\section{Background}

More than 800 women worldwide die each day from pregnancy and childbirth-related complications [1] with 99\% of these occurring in low and lower middle-income countries [2-5]. South Asian and sub-Saharan African countries bear the highest burden of maternal death [6]. The widest level of disparity in maternal mortality is between low/lower middle, and high-income countries. The lifetime risk of maternal death in high income countries is one in 3700, compared with one in 160 live births in low income countries [7]. It has been estimated that $16 \%$ to $33 \%$ of all maternal mortality may be avoided by preventing complications through the provision of skilled personnel at birth [8].

Quality care throughout pregnancy and childbirth is associated with good maternal and infant outcomes [9]. Antenatal care uptake in rural settings is dependent on social and cultural factors [10]. Increased access to antenatal care, provision of skilled birth attendants and pregnancy care awareness programs at the local level contribute to safer pregnancies and childbirth [11]. In rural families, money, food and other logistics have all been seen to have an important influence on maternal health outcomes [12]. For instance, in Nepal, pregnancy and childbirth are considered to be the domain of women [13]. Although mothers-in-law commonly make decisions on pregnancy-related issues [14], permission must be sought from the male head of the household for any costs associated with seeking pregnancy care. As it is very uncommon for men to take an interest in pregnancy-related care issues, the resulting lack of communication limits women's access to pregnancy care [15]. Gender disparity and discrimination is common in South Asian countries like Nepal and is particularly prevalent in rural settings [16].

Health care interventions are often limited to a rigid and structured operational framework rather than being designed to meet the socio-cultural and economic realities of the communities they serve [17]. National programs and strategies often fail to consider the hardship imposed by distance and lack of infrastructure that is peculiar to people living within rural settings [18]. Poor social status among women in South Asian countries is a great contributor to lack of family planning and a rising population growth. This "feminisation of poverty" in the region is a fundamental anomaly that has impaired societal development [19]. Engaging local people to educate and mobilise the community has the capacity to provide multidimensional benefits [20] such as: helping to modify practices [21], encouraging a sense of community [22], and emboldening the identification of local methods to address problems [23].

Changing people's attitudes is required, but the best method for doing this is unclear. This systematic review aimed to compare the overall effect of different approaches to community participation in maternal health care education compared with health service or control/ standard care interventions, on important maternal health outcomes. Furthermore, this systematic review aimed to examine which interventions promote husband, family and community awareness and involvement in maternal health care and result in better maternal health care-seeking and utilisation of maternal health services for improved maternal health outcomes in rural South Asian countries.

The overall objective of this systematic review was to compare the effectiveness of interventions to promote family and community participation in maternal health care against standard health care and health service led programs on the outcomes: indicators of maternal health care knowledge improvement, maternal health care utilisation (antenatal care, facility birth, skilled birth attendant use), and maternal mortality in rural-remote regions of the South Asian countries, Bangladesh, India, Nepal, and Pakistan.

\section{Methods}

\section{Search strategy and selection criteria}

Database searches were initially conducted in November 2015 and updated in June 2017. No restriction was placed on language or year of publication (refer Medline search strategy, Additional file 1). Hand searches were conducted on the reference lists of included studies.

This systematic review and meta-analysis were conducted according to a prospectively registered protocol (PROSPERO CRD42016033201) dated 14 January, 2016 and reported as per the Preferred Reporting in Systematic Reviews and Meta analyses (PRISMA) Guidelines [24].

We included cluster randomised trials or randomised trials evaluating different community health promotion interventions compared against each other, or against control or health service-based interventions, involving women, men, family and community members living within rural areas of Nepal, Bangladesh, India and Pakistan. We excluded studies of urban populations, and middle to high-income countries. Primary outcomes included: indicators of improved knowledge among women of childbearing age, their husbands/partners, family and community members, skilled provider attendance at birth (formal provider, traditional birth assistant), delivery at a health care facility and maternal mortality. The secondary outcome was male involvement in supporting access to maternal health care provision defined as; the supportive role played by men particularly husband, father-in-law and others with decision making capacity to access care during pregnancy, childbirth and postnatal period [25]. Traditional birth assistant (TBA) is defined as; "a person normally a female, who assists 
mothers during childbirth and who initially learns her skills delivering babies by herself or by working with another more experienced TBA" [26].

Two authors (BBS, LJ) independently extracted data from the full-text articles using the Covidence program [27]. Any differences in opinion were resolved by consensus. Data were cross-checked for accuracy by both authors. Reviewers (BBS, LJ) independently assessed the risk of bias (low, high, or unclear) (refer Additional file 2) of all included trials using the Cochrane 'Risk of bias' tool and evidence quality was assessed using GRADE $[28,29]$. Any disagreements were resolved by discussion until consensus was reached. Data were analysed using Review Manager Version 5.3. Meta-analyses were performed using fixed-effects modelling, random-effects modelling was substituted in situations where heterogeneity $\left(\mathrm{I}^{2}\right)$ was greater than $20 \%$. Publication bias was assessed by visual inspection of funnel plots and incorporated in GRADE quality assessment [30]. Results for dichotomous data were presented as risk ratios (RR) with 95\% confidence intervals (CI) [30. Continuous data were reported using mean difference (MD) or standardised mean difference (SMD) where appropriate [30]. All trials randomised the intervention at village or district level. To avoid unit of analysis error caused by clustering, the intra-cluster coefficient (ICC) was used to calculate the effective sample size of both intervention and control arms which was then used to adjust the standard error for all analyses [30]. Where not provided in the study, ICC estimates were imputed from other sources [31]. Data were entered into the analysis as inverse ratios $(\log \mathrm{OR}$ or $\operatorname{logRR})$ and adjusted standard errors. Sensitivity analyses were conducted including only trials of moderate quality or above [30].

In order to consider a study as overall having low risk of bias we defined that it had to have none of the domains considered as high risk of bias and at least four (not counting 'Other biases') considered as low risk of bias, and two of these must include 'random sequence generation' and 'incomplete outcome data' as the domains most likely to influence overall measurements of effect.

\section{Results}

We identified 5440 citations in our search strategy, removed 525 duplicates and screened title and abstracts of 4915 (refer to Fig. 1 'Study selection'), resulting in 115 articles which we assessed in full text against the inclusion and exclusion criteria. Of the 45 articles remaining, we excluded nine studies that did not report any of the stated review outcomes, 15 contained insufficient information to make a judgement on whether they should be included or excluded, and six related to registered trial protocols awaiting publication of results. Of the 15 remaining publications there were only 11 independent studies as four studies had appeared in more than one publication.

Pregnant women and women of reproductive age (15 to 49 years or $<50$ years) were most commonly targeted (9 studies), in addition to their in-laws ( 1 study), community members of any age ( 2 studies), and both women and men of reproductive age (1 study) (Tables 1 and 2). All studies were from rural and remote areas of low or lower middle income South Asian countries sharing a similar burden of maternal morbidity and mortality, including India (3 studies), Nepal (2 studies), Bangladesh (4 studies) and Pakistan (2 studies). Population sample sizes ranged from 1058 to 29,889 pregnant women, communities (health units, rural unions) or live births.

Eleven studies randomly allocated communities to community interventions; 10 studies compared a community intervention to a standard health care or control group (Table 1), and one (Acharya) compared different types of community intervention combinations at village or district level (Table 2). Two studies (Baqui, Midhet) assessed a community intervention against standard care or control group and provided data for comparisons between different community interventions. Four studies (Acharya, Baqui, Bhutta, Sharma) involved the use of community members to mobilise their community to take part in maternal health education and take practical steps to improve maternal health care within the community. Baqui compared the use of both male and female community mobilisers with the use of female mobilisers only. Darmstadt used community health workers to deliver a community education intervention. Six studies (list studies) involved the participation of community women in maternal health education groups. The intervention periods occurred over approximately 2 years.

In addition to examining the results for each outcome, all community interventions were compared against:

a) standard care or control (10 studies) (Table 1), and;

b) other community interventions (3 studies) (Table 2);

We identified the following post-hoc Community intervention subgroups:

1) Use of community mobilisers to deliver maternal health education;

a. Home care by male and female mobilisers.

b. Community care by female mobilisers.

2) Community education by health care workers.

3) Women's maternal health care education groups.

4) Women's and men's maternal health care education groups. 


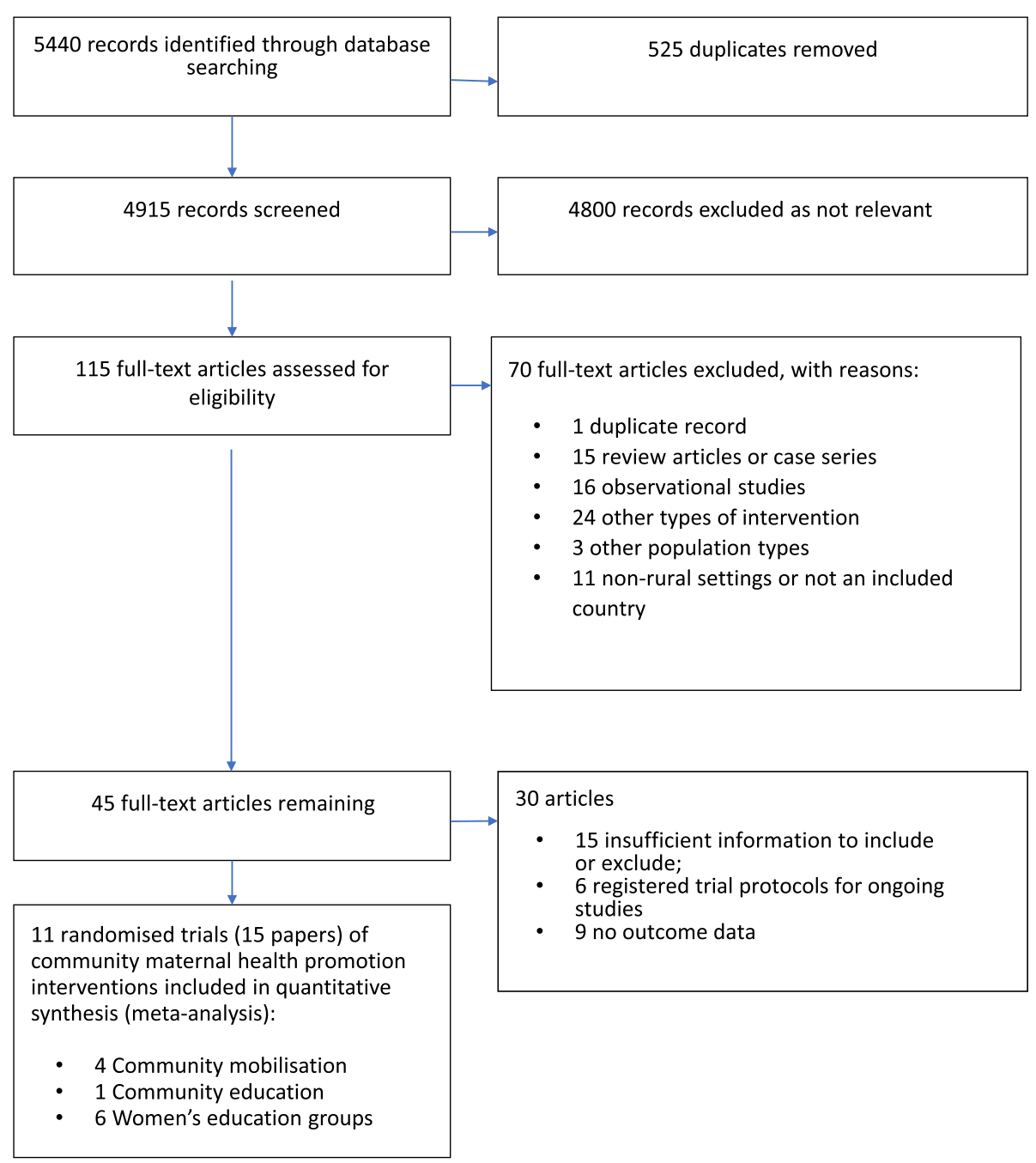

Fig. 1 Study selection

As the design of cluster randomised studies is likely to have made it difficult to provide blinded participation and assessment, we rated all studies not blinded or not reporting blinding, as unclear risk performance and detection bias. The main domains of interest for these study designs were random sequence generation, allocation concealment, incomplete outcome data and subjective reporting.

Overall, six studies were assessed as low risk of bias, two (Tripathy 2010, 2016) as unclear risk, and three (Azad, Baqui, Midhet) as high risk. Azad, Baqui and Midhet reported $>10 \%$ losses of population outcome data to the final analysis over the study follow-up period. Although population outcome data losses at follow-up were $<10 \%$, Tripathy 2010 excluded twice as many women and live births in the control group (169 women, 171 births from a total of 9260 births; $1.8 \%$ losses) compared with that of the intervention group (83 women, 84 births from a total of 9770 births; $0.9 \%$ ) due to deaths, stillbirths and migration. Similarly, Tripathy 2016 excluded a greater percentage of population outcome data from the final analysis in the intervention group (6238 women from 82,$702 ; 7.6 \%)$ compared with the control group (28 women from 73,817; 0.04\%).

The individual results of all included studies are shown in Tables 1 and 2.

Two studies (Darmstadt, Sharma) reported improved mean knowledge scores among women of childbearing age, their partners, and family and community members. There was a small improvement in knowledge of maternal danger signs RR 1.40 (95\% CI 1.12, 1.75) in receiving community education intervention by community health workers compared with a control group, whilst Sharma demonstrated improvement in maternal health care knowledge among community members receiving health care messages in a community singing intervention compared with control. Post-intervention, knowledge doubled in the intervention group from a mean of 11.60/36 
Table 1 Characteristics of studies. Community interventions versus health services, standard care or control

\begin{tabular}{|c|c|c|c|c|c|c|c|c|c|}
\hline $\begin{array}{l}\text { Study/Year } \\
\text { published } \\
\text { Country } \\
\text { Duration of } \\
\text { intervention }\end{array}$ & Design & Population & $n$ & $\begin{array}{l}\text { Risk of } \\
\text { Bias a }\end{array}$ & Intervention & Control & Outcome & OR & RR \\
\hline \multirow[t]{9}{*}{$\begin{array}{l}\text { Azad [42] } \\
\text { Bangladesh } \\
2005 \text { to } \\
2007\end{array}$} & \multirow[t]{9}{*}{$\begin{array}{l}\text { Cluster RCT } \\
18 \text { clusters }\end{array}$} & \multirow[t]{9}{*}{$\begin{array}{l}\text { Women aged } 15 \\
\text { to } 49 \text { years having } \\
\text { given birth during } \\
\text { the study period }\end{array}$} & \multirow[t]{9}{*}{29,889} & \multirow{9}{*}{$\begin{array}{l}\text { 1. Low } \\
2 . \\
\text { Unclear } \\
3 . \\
\text { Unclear } \\
\text { 4. Low } \\
\text { 5.High } \\
\text { 6.Low } \\
\text { 7.Low } \\
\text { Overall: } \\
\text { High }\end{array}$} & \multirow[t]{9}{*}{$\begin{array}{l}\text { Women's education } \\
\text { groups plus health } \\
\text { system } \\
\text { improvement }\end{array}$} & \multirow[t]{9}{*}{$\begin{array}{l}\text { Control } \\
\text { (health } \\
\text { system } \\
\text { improvement) }\end{array}$} & $\begin{array}{l}\text { Antenatal care } \\
\text { any }\end{array}$ & $\begin{array}{l}0.78[0.51 \\
1.19]^{*}\end{array}$ & $\begin{array}{l}0.91[0.76, \\
1.09]^{* *}\end{array}$ \\
\hline & & & & & & & $\geq 4$ visits & $\begin{array}{l}0.15[0.06, \\
0.40]^{*}\end{array}$ & $\begin{array}{l}0.79[0.46, \\
1.37]^{* * * *}\end{array}$ \\
\hline & & & & & & & $\begin{array}{l}\text { Skilled birth } \\
\text { attendant } \\
\text { formal provider }\end{array}$ & $\begin{array}{l}0.45[0.19 \\
1.11]^{*}\end{array}$ & $\begin{array}{l}0.90[0.72, \\
1.14]^{* *}\end{array}$ \\
\hline & & & & & & & $\begin{array}{l}\text { Delivery at a } \\
\text { health care } \\
\text { facility }\end{array}$ & $\begin{array}{l}0.75[0.62, \\
0.89]^{* * *}\end{array}$ & $\begin{array}{l}0.97[0.77, \\
1.24]^{* *}\end{array}$ \\
\hline & & & & & & & $\begin{array}{l}\text { Maternal } \\
\text { deaths }\end{array}$ & & \\
\hline & & & & & & & 2 years & - & $\begin{array}{l}1.80[1.2 \\
3.17]^{*}\end{array}$ \\
\hline & & & & & & & $\begin{array}{l}3 \text { years } \\
\text { ( } \mathrm{N}=\text { number of } \\
\text { live births for } \\
\text { all outcomes } \\
\text { above) }\end{array}$ & - & $\begin{array}{l}1.67[1.00, \\
2.79]^{*}\end{array}$ \\
\hline & & & & & & & $\begin{array}{l}\text { MMR (over } \\
3 \text { years) }\end{array}$ & $\begin{array}{l}388.9 \text { vs. } \\
189.10\end{array}$ & $\begin{array}{l}2.02[1.11, \\
3.68]^{* *}\end{array}$ \\
\hline & & & & & & & $100,000)$ & & $\begin{array}{l}1.74[0.97, \\
3.13] \#\end{array}$ \\
\hline \multirow[t]{5}{*}{$\begin{array}{l}\text { Baqui [49] } \\
\text { Bangladesh } \\
2003 \text { to } \\
2006\end{array}$} & \multirow[t]{5}{*}{$\begin{array}{l}\text { Cluster RCT } \\
24 \text { clusters }\end{array}$} & \multirow[t]{5}{*}{$\begin{array}{l}\text { All married } \\
\text { women of } \\
\text { reproductive age } \\
\text { (15-49 years) }\end{array}$} & \multirow[t]{5}{*}{5110} & \multirow{5}{*}{$\begin{array}{l}\text { 1.Low } \\
\text { 2.Low } \\
\text { 3.Unclear } \\
\text { 4.Low } \\
\text { 5.High } \\
\text { 6.Low } \\
\text { 7.Low } \\
\text { Overall: } \\
\text { High }\end{array}$} & $\begin{array}{l}\text { Community } \\
\text { mobilisation: } \\
\text { home care by } \\
\text { female mobilisers } \\
\text { visiting every } \\
8 \text { months }\end{array}$ & control & $\begin{array}{l}\text { Antenatal care } \\
\text { Any } \\
(\mathrm{N}=\text { number of } \\
\text { live births) }\end{array}$ & $\begin{array}{l}1.70[1.07, \\
2.68]^{*}\end{array}$ & $\begin{array}{l}1.13[0.93, \\
1.36]^{*}\end{array}$ \\
\hline & & & & & Community & control & Antenatal care & & \\
\hline & & & & & $\begin{array}{l}\text { Community care by } \\
\text { male mobilisers } \\
\text { visiting every } \\
10 \text { months and } \\
\text { female mobilisers } \\
\text { visiting every } \\
4 \text { months }\end{array}$ & & $\begin{array}{l}\text { Any } \\
\text { ( } \mathrm{N}=\text { number of } \\
\text { live births) }\end{array}$ & $\begin{array}{l}2.67[1.70 \\
4.21]^{*}\end{array}$ & $\begin{array}{l}1.47[1.21, \\
1.70]^{*}\end{array}$ \\
\hline & & & & & Community & control & Antenatal care & & \\
\hline & & & & & $\begin{array}{l}\text { community and } \\
\text { home care }\end{array}$ & & $\begin{array}{l}\text { Any } \\
\text { ( } \mathrm{N}=\text { number of } \\
\text { live births) }\end{array}$ & $\begin{array}{l}2.13[1.33 \\
3.39]^{*}\end{array}$ & $\begin{array}{l}1.37[1.15, \\
1.63]^{*}\end{array}$ \\
\hline \multirow{4}{*}{$\begin{array}{l}\text { Bhutta [50] } \\
\text { Pakistan } \\
2006 \text { to } \\
2008\end{array}$} & \multirow{4}{*}{$\begin{array}{l}\text { Cluster RCT } \\
16 \text { clusters }\end{array}$} & \multirow{4}{*}{$\begin{array}{l}\text { Pregnant women } \\
\text { and women of } \\
\text { reproductive age } \\
\text { (15-49 years) }\end{array}$} & \multirow[t]{4}{*}{4474} & \multirow{4}{*}{$\begin{array}{l}\text { 1.Low } \\
\text { 2.Unclear } \\
\text { 3.Unclear } \\
\text { 4.Low } \\
\text { 5.Low } \\
\text { 6.Low } \\
\text { 7.Low } \\
\text { Overall: } \\
\text { Low }\end{array}$} & \multirow{4}{*}{$\begin{array}{l}\text { Community } \\
\text { mobilisation }\end{array}$} & \multirow{4}{*}{$\begin{array}{l}\text { Healthcare } \\
\text { workers } \\
\text { (standard } \\
\text { healthcare) }\end{array}$} & Antenatal care & & \\
\hline & & & & & & & Any & $\begin{array}{l}1.64[1.03 \\
2.62]^{*}\end{array}$ & $\begin{array}{l}1.20[1.01, \\
1.42]^{*}\end{array}$ \\
\hline & & & & & & & $\geq 4$ visits & $\begin{array}{l}1.51[0.79, \\
2.88]^{*}\end{array}$ & $\begin{array}{l}1.44[0.75, \\
2.77]^{*}\end{array}$ \\
\hline & & & & & & & $\begin{array}{l}\text { Delivery at a } \\
\text { health care } \\
\text { facility (any) } \\
\text { ( } \mathrm{N}=\text { number of } \\
\text { women for all } \\
\text { outcomes) }\end{array}$ & $\begin{array}{l}1.53[1.36 \\
1.72]^{* * *}\end{array}$ & $\begin{array}{l}1.24[1.17, \\
1.32]^{* * *}\end{array}$ \\
\hline
\end{tabular}


Table 1 Characteristics of studies. Community interventions versus health services, standard care or control (Continued)

\begin{tabular}{|c|c|c|c|c|c|c|c|c|c|c|}
\hline $\begin{array}{l}\text { Study/Year } \\
\text { published } \\
\text { Country } \\
\text { Duration of } \\
\text { intervention }\end{array}$ & Design & Population & $n$ & $\begin{array}{l}\text { Risk of } \\
\text { Bias }^{a}\end{array}$ & Intervention & Control & Outcome & OR & & RR \\
\hline \multirow{9}{*}{$\begin{array}{l}\text { Darmstadt } \\
\text { [51] } \\
\text { Bangladesh } \\
2004 \text { to } \\
2006\end{array}$} & \multirow{9}{*}{$\begin{array}{l}\text { Cluster } \\
\text { randomised } \\
\text { trial } \\
12 \text { clusters }\end{array}$} & \multirow{9}{*}{$\begin{array}{l}\text { All married } \\
\text { women of } \\
\text { reproductive age } \\
\text { (15-49 years) }\end{array}$} & \multirow[t]{9}{*}{3491} & \multirow{9}{*}{$\begin{array}{l}\text { 1.Low } \\
\text { 2.Unclear } \\
\text { 3.Unclear } \\
\text { 4.Low } \\
\text { 5.Low } \\
\text { 6.Low } \\
\text { 7.Low } \\
\text { Overall: } \\
\text { Low }\end{array}$} & \multirow{9}{*}{$\begin{array}{l}\text { Community } \\
\text { education by } \\
\text { community health } \\
\text { care workers }\end{array}$} & \multirow[t]{9}{*}{ Control } & Antenatal care & & & \\
\hline & & & & & & & Any & \multicolumn{2}{|c|}{$\begin{array}{l}2.29[1.34 \\
3.91]^{*}\end{array}$} & $\begin{array}{l}1.40[1.12, \\
1.75]^{*}\end{array}$ \\
\hline & & & & & & & $\begin{array}{l}\text { Skilled birth } \\
\text { attendant }\end{array}$ & \multicolumn{2}{|c|}{$\begin{array}{l}\text { Not } \\
\text { reported }\end{array}$} & $\begin{array}{l}\text { Not } \\
\text { reported }\end{array}$ \\
\hline & & & & & & & $\begin{array}{l}\text { Delivery at } \\
\text { health care } \\
\text { facility } \\
\text { ( } \mathrm{N}=\text { number of } \\
\text { women for all } \\
\text { outcomes) }\end{array}$ & \multicolumn{2}{|c|}{$\begin{array}{l}1.20[0.62, \\
2.29]^{* * *}\end{array}$} & $\begin{array}{l}1.19[0.63, \\
2.27]^{* * *}\end{array}$ \\
\hline & & & & & & & \multirow{2}{*}{$\begin{array}{l}\text { Maternal } \\
\text { danger sign } \\
\text { knowledge } \\
\text { score: }\end{array}$} & \multicolumn{2}{|c|}{$\begin{array}{l}\text { Mean score } \\
\text { Intervention }\end{array}$} & $\begin{array}{l}\text { Mean score } \\
\text { Control }\end{array}$ \\
\hline & & & & & & & & Pre & Post & Pre Post \\
\hline & & & & & & & $\begin{array}{l}\text { a. Antenatal } \\
{[0-10]}\end{array}$ & 1.0 & 2.9 & $1.1 \quad 2.2$ \\
\hline & & & & & & & $\begin{array}{l}\text { b. Labor/ } \\
\text { delivery [0- } \\
11]\end{array}$ & 1.1 & 2.4 & $1.2 \quad 1.9$ \\
\hline & & & & & & & $\begin{array}{l}\text { C. } \\
\text { Postpartum } \\
{[0-9]}\end{array}$ & 1.0 & 2.5 & $1.0 \quad 2.5$ \\
\hline \multirow{6}{*}{$\begin{array}{l}\text { Fottrell [52] } \\
\text { Bangladesh } \\
2005 \text { to } \\
2007\end{array}$} & \multirow{6}{*}{$\begin{array}{l}\text { Cluster } \\
\text { randomised } \\
\text { trial } \\
18 \text { Clusters }\end{array}$} & \multirow{6}{*}{$\begin{array}{l}\text { Ever-married } \\
\text { women of } \\
\text { reproductive age } \\
\text { (15-49 years) who } \\
\text { were permanent } \\
\text { residents including } \\
\text { in-laws and ado- } \\
\text { lescent girls }\end{array}$} & \multirow[t]{6}{*}{17,940} & \multirow{6}{*}{$\begin{array}{l}\text { 1.Low } \\
\text { 2.Unclear } \\
\text { 3.Unclear } \\
\text { 4.Low } \\
\text { 5.Low } \\
\text { 6.Low } \\
\text { 7.Unclear } \\
\text { Overall: } \\
\text { Low }\end{array}$} & \multirow{6}{*}{$\begin{array}{l}\text { Women's education } \\
\text { groups plus health } \\
\text { system } \\
\text { improvement }\end{array}$} & \multirow{6}{*}{$\begin{array}{l}\text { Control } \\
\text { (health } \\
\text { system } \\
\text { improvement) }\end{array}$} & Antenatal care & & & \\
\hline & & & & & & & $\geq 4$ visits & \multicolumn{2}{|c|}{$\begin{array}{l}1.37[0.99- \\
1.88]^{* *}\end{array}$} & $\begin{array}{l}1.28[0.13, \\
12.11]^{*}\end{array}$ \\
\hline & & & & & & & $\begin{array}{l}\text { Skilled birth } \\
\text { attendant }\end{array}$ & \multicolumn{2}{|c|}{$\begin{array}{l}0.54[0.38 \\
0.78]^{* *}\end{array}$} & $\begin{array}{l}0.92[0.71 \\
1.20]^{*}\end{array}$ \\
\hline & & & & & & & $\begin{array}{l}\text { Delivery at } \\
\text { health care } \\
\text { facility (any) } \\
\text { ( } \mathrm{N}=\text { number of } \\
\text { births for } \\
\text { above } \\
\text { outcomes) }\end{array}$ & \multicolumn{2}{|c|}{$\begin{array}{l}1.05[0.88 \\
1.25]^{* *}\end{array}$} & $\begin{array}{l}0.94[0.55 \\
1.59]^{* * *}\end{array}$ \\
\hline & & & & & & & $\begin{array}{l}\text { Maternal } \\
\text { deaths } \\
2 \text { years } \\
\text { ( } \mathrm{N}=\text { number of } \\
\text { live births) }\end{array}$ & \multicolumn{2}{|l|}{-} & $\begin{array}{l}0.59[0.30, \\
1.18]^{*}\end{array}$ \\
\hline & & & & & & & $\begin{array}{l}\text { MMR (over } \\
2 \text { years) } \\
\text { (per 100,000 } \\
\text { live births) }\end{array}$ & \multicolumn{2}{|c|}{$\begin{array}{l}153.4 \text { vs. } \\
276.10\end{array}$} & $\begin{array}{l}0.74[0.34 \\
1.64]^{* *}\end{array}$ \\
\hline \multirow{4}{*}{$\begin{array}{l}\text { Midhet** } \\
\text { [53] } \\
\text { Pakistan } \\
\text { 1998-2002 }\end{array}$} & Cluster & Women and men & 2564 & 1.Low & Women's and men's & Control & Antenatal care & & & \\
\hline & $\begin{array}{l}24 \text { clusters } \\
\text { trial }\end{array}$ & $\begin{array}{l}\text { of reproauctive } \\
\text { age } \\
\text { (all ever-married }\end{array}$ & & $\begin{array}{l}\text { 3.Unclear } \\
\text { 4.Low }\end{array}$ & & & Any & $\begin{array}{l}2.83 \\
5.00\end{array}$ & 1.60, & $\begin{array}{l}1.35[0.81 \\
2.25]^{*}\end{array}$ \\
\hline & & $\begin{array}{l}\text { women under } \\
50 \text { years of age) }\end{array}$ & & $\begin{array}{l}\text { 5.High } \\
\text { 6.Unclear } \\
\text { 7.Low } \\
\text { Overall: } \\
\text { High }\end{array}$ & & & $\begin{array}{l}\text { Delivery at } \\
\text { health care } \\
\text { facility (any) } \\
\text { ( } \mathrm{N}=\text { number of } \\
\text { pregnant } \\
\text { women for all } \\
\text { outcomes) }\end{array}$ & $\begin{array}{l}1.3[ \\
2.7]^{*}\end{array}$ & & $\begin{array}{l}1.28[0.84 \\
1.96]^{* * *}\end{array}$ \\
\hline & & & & & Women's education & Control & Antenatal care & & & \\
\hline
\end{tabular}


Table 1 Characteristics of studies. Community interventions versus health services, standard care or control (Continued)

\begin{tabular}{|c|c|c|c|c|c|c|c|c|c|}
\hline $\begin{array}{l}\text { Study/Year } \\
\text { published } \\
\text { Country } \\
\text { Duration of } \\
\text { intervention }\end{array}$ & Design & Population & $n$ & $\begin{array}{l}\text { Risk of } \\
\text { Bias }^{\text {a }}\end{array}$ & Intervention & Control & Outcome & OR & RR \\
\hline & & & & & groups & & Any & $\begin{array}{l}2.45[1.40 \\
4.30]^{* *}\end{array}$ & $\begin{array}{l}1.32[0.79, \\
2.20]^{*}\end{array}$ \\
\hline & & & & & & & $\begin{array}{l}\text { Delivery at a } \\
\text { health care } \\
\text { facility } \\
\text { ( } \mathrm{N}=\text { number of } \\
\text { pregnant } \\
\text { women for all } \\
\text { outcomes) }\end{array}$ & $\begin{array}{l}1.3[0.7, \\
2.5]^{* *}\end{array}$ & $\begin{array}{l}1.32[0.86 \\
2.02]^{* * *}\end{array}$ \\
\hline & & & & & Both interventions & Control & Antenatal care & & \\
\hline & & & & & $\begin{array}{l}\text { combined } \\
\text { (women's education } \\
\text { groups and }\end{array}$ & & Any & $\begin{array}{l}1.38[0.82, \\
1.34]^{*}\end{array}$ & $\begin{array}{l}1.33[0.84 \\
2.10]^{*}\end{array}$ \\
\hline & & & & & $\begin{array}{l}\text { women's and men's } \\
\text { education groups) }\end{array}$ & & $\begin{array}{l}\text { Delivery at } \\
\text { health care } \\
\text { facility (any) } \\
\text { ( } \mathrm{N}=\text { number of } \\
\text { pregnant } \\
\text { women for all } \\
\text { outcomes) }\end{array}$ & $\begin{array}{l}1.46[0.99 \\
2.15]^{* * *}\end{array}$ & $\begin{array}{l}1.43[0.99 \\
2.07]^{* * *}\end{array}$ \\
\hline \multirow{8}{*}{$\begin{array}{l}\text { Osrin [54] } \\
\text { Nepal } \\
\text { 1998-2000 }\end{array}$} & \multirow{8}{*}{$\begin{array}{l}\text { Randomised } \\
\text { trial } \\
24 \text { Clusters }\end{array}$} & \multirow{8}{*}{$\begin{array}{l}\text { Women (aged 15- } \\
49 \text { years) and key } \\
\text { members of the } \\
\text { community in } \\
\text { improving } \\
\text { perinatal health } \\
\text { outcomes }\end{array}$} & \multirow[t]{8}{*}{4241} & \multirow{8}{*}{$\begin{array}{l}\text { 1.Low } \\
\text { 2.Unclear } \\
\text { 3.Unclear } \\
\text { 4.Low } \\
\text { 5.Low } \\
\text { 6.Low } \\
\text { 7.Low } \\
\text { Overall: } \\
\text { Low }\end{array}$} & \multirow{8}{*}{$\begin{array}{l}\text { Women's education } \\
\text { groups }\end{array}$} & \multirow[t]{8}{*}{ Control } & Antenatal care & & \\
\hline & & & & & & & $\begin{array}{l}\text { Any } \\
\text { ( } N=\text { number of } \\
\text { pregnancies) } \\
\text { Skilled birth } \\
\text { attendant }\end{array}$ & $\begin{array}{l}2.82[1.41 \\
5.62]^{* *}\end{array}$ & $\begin{array}{l}1.32[1.08 \\
1.60]^{*}\end{array}$ \\
\hline & & & & & & & Any & $\begin{array}{l}2.50[1.51 \\
4.16]^{*}\end{array}$ & $\begin{array}{l}2.26[1.43 \\
3.57]^{*}\end{array}$ \\
\hline & & & & & & & $\begin{array}{l}\text { formal health } \\
\text { provider } \\
\text { (doctor or } \\
\text { nurse) }\end{array}$ & $\begin{array}{l}3.13[1.62 \\
6.03]^{* *}\end{array}$ & $\begin{array}{l}2.96[1.50 \\
5.84]^{*}\end{array}$ \\
\hline & & & & & & & $\begin{array}{l}\text { Traditional } \\
\text { birth assistant } \\
\text { ( } \mathrm{N}=\text { number of } \\
\text { deliveries) }\end{array}$ & $\begin{array}{l}1.70[0.93 \\
3.11]^{* *}\end{array}$ & $\begin{array}{l}1.71[0.89 \\
3.31]^{*}\end{array}$ \\
\hline & & & & & & & $\begin{array}{l}\text { Delivery at } \\
\text { health care } \\
\text { facility }\end{array}$ & $\begin{array}{l}3.54[1.56 \\
8.05]^{* *}\end{array}$ & $\begin{array}{l}3.38[2.57 \\
4.45]^{* * *}\end{array}$ \\
\hline & & & & & & & $\begin{array}{l}\text { Maternal } \\
\text { deaths } \\
2 \text { years }\end{array}$ & & $\begin{array}{l}0.18[0.14 \\
0.24]^{*}\end{array}$ \\
\hline & & & & & & & $\begin{array}{l}\text { MMR (over } \\
3 \text { years) } \\
\text { (per 100,000 } \\
\text { live births) }\end{array}$ & 69 vs 341 & $\begin{array}{l}0.22[0.05 \\
0.90]^{* *}\end{array}$ \\
\hline \multirow[t]{3}{*}{$\begin{array}{l}\text { Sharma } \\
2018 \text { [34] } \\
\text { Nepal }\end{array}$} & \multirow[t]{3}{*}{$\begin{array}{l}\text { Pre and } \\
\text { post-test } \\
\text { randomised }\end{array}$} & \multirow[t]{3}{*}{$\begin{array}{l}\text { All community } \\
\text { members (all ages } \\
\text { eligible) }\end{array}$} & \multirow[t]{3}{*}{1572} & \multirow{3}{*}{$\begin{array}{l}\text { 1.Low } \\
\text { 2.Unclear } \\
\text { 3.Unclear } \\
\text { 4.Low } \\
\text { 5.Low } \\
\text { 6.Low } \\
\text { 7.Unclear } \\
\text { Overall: } \\
\text { Low }\end{array}$} & \multirow{3}{*}{$\begin{array}{l}\text { Community } \\
\text { mobilisation: } \\
\text { Community singing } \\
\text { to deliver } \\
\text { healthcare } \\
\text { messages }\end{array}$} & \multirow[t]{3}{*}{ Control } & $\begin{array}{l}\text { 1. Mean } \\
\text { change in } \\
\text { knowledge } \\
\text { scores (se): }\end{array}$ & & \\
\hline & & & & & & & $\begin{array}{l}\text { a. Importance } \\
\text { of antenatal } \\
\text { examination } \\
\text { (out of } 7 \text { ) }\end{array}$ & $\begin{array}{l}2 \cdot 12[0 \cdot 06] \\
\text { Vs } 4.89 \\
{[0.06]}\end{array}$ & \\
\hline & & & & & & & b. Importance & $3.71[0.07]$ & $0.12[-0.22$ \\
\hline
\end{tabular}


Table 1 Characteristics of studies. Community interventions versus health services, standard care or control (Continued)

\begin{tabular}{|c|c|c|c|c|c|c|c|c|c|}
\hline $\begin{array}{l}\text { Study/Year } \\
\text { published } \\
\text { Country } \\
\text { Duration of } \\
\text { intervention }\end{array}$ & Design & Population & $\mathrm{n}$ & $\begin{array}{l}\text { Risk of } \\
\text { Bias }^{\text {a }}\end{array}$ & Intervention & Control & Outcome & OR & RR \\
\hline & & & & & & & $\begin{array}{l}\text { of } \\
\text { supplementary } \\
\text { diet and rest } \\
\text { during } \\
\text { pregnancy (out } \\
\text { of 9) }\end{array}$ & $\begin{array}{l}\text { vs } 6.84 \\
{[0.06]}\end{array}$ & $0.46]$ \\
\hline & & & & & & & $\begin{array}{l}\text { c. Importance } \\
\text { of delivery care } \\
\text { (out of 12) }\end{array}$ & $\begin{array}{l}2 \cdot 95[0.08] \\
\text { vs } 5.09 \\
{[0.07]}\end{array}$ & \\
\hline & & & & & & & $\begin{array}{l}\text { d. Importance } \\
\text { of childbirth } \\
\text { planning (out } \\
\text { of } 8 \text { ) }\end{array}$ & $\begin{array}{l}2 \cdot 81[0.08] \\
\text { Vs } 5 \cdot 50 \\
{[0.06]}\end{array}$ & $\begin{array}{l}-0.71[-1.3, \\
-0.11]\end{array}$ \\
\hline & & & & & & & $\begin{array}{l}\text { e. Overall } \\
\text { knowledge } \\
\text { (out of 36) }\end{array}$ & $\begin{array}{l}11 \cdot 60[0 \cdot 24] \\
\text { vs } 22 \cdot 33 \\
{[0.18]}\end{array}$ & $\begin{array}{l}-1.02[-2, \\
-0.03]\end{array}$ \\
\hline \multirow{13}{*}{$\begin{array}{l}\text { Tripathy } \\
2010 \text { [55] } \\
\text { India } \\
2005 \text { to } \\
2008\end{array}$} & \multirow{13}{*}{$\begin{array}{l}\text { Cluster RCT } \\
18 \text { clusters }\end{array}$} & \multirow{13}{*}{$\begin{array}{l}\text { Women of } \\
\text { reproductive age } \\
\text { (15-49 years) }\end{array}$} & \multirow[t]{13}{*}{17,335} & \multirow{13}{*}{$\begin{array}{l}\text { 1.Low } \\
\text { 2.Unclear } \\
\text { 3.Unclear } \\
\text { 4.Low } \\
\text { 5.Unclear } \\
\text { 6.Low } \\
\text { 7.Unclear } \\
\text { Overall: } \\
\text { Unclear }\end{array}$} & \multirow{13}{*}{$\begin{array}{l}\text { Women's education } \\
\text { groups plus health } \\
\text { system } \\
\text { improvement }\end{array}$} & \multirow{13}{*}{$\begin{array}{l}\text { Control } \\
\text { (health } \\
\text { system } \\
\text { improvement) }\end{array}$} & \multicolumn{3}{|l|}{ Antenatal care } \\
\hline & & & & & & & \multirow[t]{2}{*}{ Any } & $\begin{array}{l}0.97[0.48 \\
1.97]^{* *}\end{array}$ & \multirow[t]{2}{*}{$\begin{array}{l}1.11[0.99, \\
1.23]^{*}\end{array}$} \\
\hline & & & & & & & & $\begin{array}{l}1.60(0.65- \\
3.92) \#\end{array}$ & \\
\hline & & & & & & & $>3$ visits & $\begin{array}{l}0.68[0.37, \\
1.24]^{* *}\end{array}$ & $\begin{array}{l}0.70[0.57, \\
0.87]^{*}\end{array}$ \\
\hline & & & & & & & \multicolumn{3}{|l|}{$\begin{array}{l}\text { Skilled birth } \\
\text { attendant }\end{array}$} \\
\hline & & & & & & & Any & $\begin{array}{l}0.52[0.37 \\
0.74]^{* *}\end{array}$ & $\begin{array}{l}0.70[0.57 \\
0.87]^{*}\end{array}$ \\
\hline & & & & & & & $\begin{array}{l}\text { Formal } \\
\text { provider }\end{array}$ & $\begin{array}{l}0.59[0.37 \\
0.94]^{*}\end{array}$ & $\begin{array}{l}0.67[0.46, \\
1.00]^{*}\end{array}$ \\
\hline & & & & & & & $\begin{array}{l}\text { Traditional } \\
\text { birth assistant }\end{array}$ & $\begin{array}{l}0.82[0.43, \\
1.56]^{*}\end{array}$ & $\begin{array}{l}0.88[0.68, \\
1.13]^{*}\end{array}$ \\
\hline & & & & & & & $\begin{array}{l}\text { Delivery at } \\
\text { health care } \\
\text { facility(any) } \\
\text { ( } N=\text { number of } \\
\text { pregnant } \\
\text { women for all } \\
\text { outcomes) }\end{array}$ & $\begin{array}{l}0.64[0.39, \\
1.04]^{* *}\end{array}$ & $\begin{array}{l}0.71[0.66 \\
0.75]^{* * * *}\end{array}$ \\
\hline & & & & & & & \multicolumn{3}{|l|}{$\begin{array}{l}\text { Maternal } \\
\text { deaths }\end{array}$} \\
\hline & & & & & & & 2 years & & $\begin{array}{l}0.82[0.51 \\
1.33]^{*}\end{array}$ \\
\hline & & & & & & & 3 years & & $\begin{array}{l}0.77[0.53, \\
1.13]^{*}\end{array}$ \\
\hline & & & & & & & $\begin{array}{l}\text { MMR (over } \\
3 \text { years) } \\
\text { (per 100,000 } \\
\text { live births) }\end{array}$ & $\begin{array}{l}517.5 \text { vs } \\
680.3\end{array}$ & $\begin{array}{l}0.70[0.46, \\
1.07]^{* *}\end{array}$ \\
\hline \multirow{3}{*}{$\begin{array}{l}\text { Tripathy } \\
2016 \text { [56] } \\
\text { India } \\
2009 \text { to } \\
2012\end{array}$} & \multirow{3}{*}{$\begin{array}{l}\text { Randomised } \\
\text { Controlled } \\
\text { Trial } \\
30 \text { clusters }\end{array}$} & \multirow{3}{*}{$\begin{array}{l}\text { Women of } \\
\text { reproductive age } \\
\text { (15-49 years) }\end{array}$} & \multirow[t]{3}{*}{7100} & \multirow{3}{*}{$\begin{array}{l}\text { 1.Low } \\
\text { 2.Unclear } \\
\text { 3.Unclear } \\
\text { 4.Low } \\
\text { 5.Unclear } \\
\text { 6.Low } \\
\text { 7. ow }\end{array}$} & \multirow{3}{*}{$\begin{array}{l}\text { Women's education } \\
\text { groups plus health } \\
\text { system } \\
\text { improvement }\end{array}$} & \multirow{3}{*}{$\begin{array}{l}\text { Control } \\
\text { (health } \\
\text { system } \\
\text { improvement) }\end{array}$} & Antenatal Care & & \\
\hline & & & & & & & Any & $\begin{array}{l}0.82[0.35 \\
1.92]^{* *}\end{array}$ & $\begin{array}{l}0.90[0.75, \\
1.07]^{*}\end{array}$ \\
\hline & & & & & & & & $\begin{array}{l}0.63[0.35- \\
1 \cdot 16] \#\end{array}$ & $\begin{array}{l}1.17[0.78, \\
1.77]^{*}\end{array}$ \\
\hline
\end{tabular}


Table 1 Characteristics of studies. Community interventions versus health services, standard care or control (Continued)

\begin{tabular}{|c|c|c|c|c|c|c|c|c|c|}
\hline $\begin{array}{l}\text { Study/Year } \\
\text { published } \\
\text { Country } \\
\text { Duration of } \\
\text { intervention }\end{array}$ & Design & Population & $n$ & $\begin{array}{l}\text { Risk of } \\
\text { Bias }^{\text {a }}\end{array}$ & Intervention & Control & Outcome & OR & RR \\
\hline & & & & \multirow[t]{4}{*}{$\begin{array}{l}\text { Overall: } \\
\text { Unclear }\end{array}$} & & & $\begin{array}{l}>=3 \text { visits } \\
(\mathrm{N}=\text { number of } \\
\text { births })\end{array}$ & $\begin{array}{l}1.08[0.58, \\
2.01]^{*}\end{array}$ & $\begin{array}{l}1.16[1.12, \\
1.20]^{* *}\end{array}$ \\
\hline & & & & & & & $\begin{array}{l}\text { Delivery at } \\
\text { health care } \\
\text { facility }\end{array}$ & $\begin{array}{l}1.23[0.58, \\
2.60]^{* *}\end{array}$ & $\begin{array}{l}0.63[0.25 \\
1.42]^{* *}\end{array}$ \\
\hline & & & & & & & $\begin{array}{l}\text { Maternal } \\
\text { deaths } \\
2 \text { years } \\
\text { ( } N=\text { number of } \\
\text { live births for } \\
\text { all of the } \\
\text { above } \\
\text { outcomes) }\end{array}$ & - & $\begin{array}{l}0.63[0.26, \\
1.55]^{*}\end{array}$ \\
\hline & & & & & & & $\begin{array}{l}\text { MMR (over } \\
2 \text { years) } \\
\text { (per 100,000 } \\
\text { live births) }\end{array}$ & $\begin{array}{l}222 \text { vs. } 349 \\
0.63[0.25 \\
1.42]^{* * *}\end{array}$ & \\
\hline
\end{tabular}

${ }^{\mathrm{a}}$ Risk of Bias tool (Cochrane)

*Adjusted estimate using outcome specific ICC

${ }^{* *}$ Adjusted estimate reported by authors - adjusted for clustering and stratification

*** Unadjusted estimate reported by authors

\#Adjusted estimate reported by authors - adjusted for clustering, stratification and baseline covariates

to $22.33 / 36$, an increase of 10.69 points $[P<0.001]$, with only a modest change in the control population $[17.48 /$ 36 to $18.26 / 36]$.

For the meta-analyses, all community interventions combined increased attendance of at least one antenatal visit compared with control by an average of $19 \%$ (RR 1.19, $95 \%$ CI 1.06 to 1.33; participants $=75,737$; studies $=8 ; \mathrm{I}^{2}=58 \%$ ) (Fig. 2). Intervention sub groups: use of community mobilisers, community care using female mobilisers, home care using both male and female mobilisers, and community education by health workers, had a similar effect (Fig. 2). However, there was no difference in effect in the number of women seeking at least one antenatal visit for community care using female mobilisers, women's education groups, and women's and men's education groups. There was no difference in the number of women attending three or more antenatal visits from any community interventions, or any intervention subgroups, compared with control (Fig. 3). Likewise, there was no difference in attendance by a person of any skill level, formal provider (doctor or nurse) or traditional birth attendant between community intervention and control groups (Fig. 4). Deliveries in health facilities were increased in women's education groups for meta-analyses of studies reporting adjusted RR 1.15 (95\% CI 1.11 to 1.20 ; participants $=36,989$; studies $=2$; $\mathrm{I}^{2}=48 \%$ ) but not in meta-analyses of studies reporting adjusted OR 1.19 (95\% CI 0.71 to 1.99; participants = 49,590; studies $=4 ; I^{2}=76 \%$ ) (Fig. 5). Estimates of RR and OR are more likely to be similar when the number of events is rare, which may explain the difference between $\mathrm{OR}$ and $\mathrm{RR}$ for antenatal care attendance as the number of events are comparatively much higher. For this reason, we have based our discussion and conclusions on RR in preference to OR. There was no difference in risk of maternal deaths at two (RR 0.63, 95\% CI 0.24 to 1.64; participants $=61,487$; studies $=5 ; 94 \%)$, and 3 years $(R R 1.11$, 95\% CI 0.52 to 2.36; participants $=48,921$; studies $=2 ; \mathrm{I}^{2}=$ $82 \%)$, between women's education groups and health service, standard care or control (Fig. 6).

No studies reported on the secondary outcome, indicators of male involvement (fathers-in-law and male partners) in maternal health care.

In addition to the varying results and evidence quality in the interventions and intervention subgroups described above, further investigation of potential contributors to heterogeneity by population (country), and outcome measurement (follow-up period 2 years vs. 3 years) subgroups showed no reduction in heterogeneity for any combination of community mobilisation interventions versus standard healthcare or control. There were insufficient data to explore the effect of these on the remaining comparisons.

\section{Discussion}

Overall, meta-analysis of all community interventions combined showed modest benefits of in terms of improving attendance of at least one antenatal care visit 
Table 2 Community participation interventions versus combined health service and community interventions or other types of community-based interventions

\begin{tabular}{|c|c|c|c|c|c|c|c|c|}
\hline $\begin{array}{l}\text { Study/Year } \\
\text { published } \\
\text { Country } \\
\text { Duration of } \\
\text { intervention }\end{array}$ & Design & Population & $\begin{array}{l}n \text { Risk of } \\
\text { Bias }^{\text {a }}\end{array}$ & $\begin{array}{l}\text { Community and Health } \\
\text { Service Intervention }\end{array}$ & $\begin{array}{l}\text { Health Service } \\
\text { Intervention }\end{array}$ & Outcome & OR & $\mathrm{RR}$ \\
\hline \multirow{14}{*}{$\begin{array}{l}\text { Acharya } \\
2015 \text { [57] } \\
\text { India } \\
2005 \text { to } \\
2011\end{array}$} & \multirow[t]{14}{*}{$\mathrm{RCT}$} & \multirow{14}{*}{$\begin{array}{l}\text { Women becoming } \\
\text { pregnant or giving } \\
\text { birth during the } \\
\text { study period } \\
\text { (average age } \\
27 \text { years) and their } \\
\text { families }\end{array}$} & \multirow{14}{*}{$\begin{array}{l}\text { 1. Low } \\
\text { 2. } \\
\text { Unclear } \\
\text { 3. } \\
\text { Unclear } \\
\text { 4. Low } \\
\text { 5. Low } \\
\text { 6. Low } \\
\text { 7. } \\
\text { Unclear } \\
\text { Overall: } \\
\text { Low }\end{array}$} & \multirow[t]{4}{*}{$\begin{array}{l}\text { Community mobilisation } \\
\text { at community level (L2) }\end{array}$} & \multirow{4}{*}{$\begin{array}{l}\text { Community } \\
\text { mobilisation at } \\
\text { community level } \\
\text { combined with } \\
\text { health care } \\
\text { messages at } \\
\text { district level (L1 } \\
\text { and L2) }\end{array}$} & $\begin{array}{l}\text { Antenatal } \\
\text { care }\end{array}$ & & \\
\hline & & & & & & Any & $1.21[0.86,1.70]^{*}$ & $\begin{array}{l}1.04 \\
{[0.97} \\
1.10]^{*}\end{array}$ \\
\hline & & & & & & $\begin{array}{l}\text { Skilled } \\
\text { birth } \\
\text { attendant }\end{array}$ & $0.90[0.56,1.43]^{*}$ & $\begin{array}{l}0.91 \\
{[0.61} \\
1.35]^{*}\end{array}$ \\
\hline & & & & & & $\begin{array}{l}\text { Delivery } \\
\text { at a } \\
\text { health } \\
\text { facility } \\
\text { ( } \mathrm{N}= \\
\text { number } \\
\text { of women } \\
\text { for all } \\
\text { outcomes) }\end{array}$ & $1.10[1.03,1.17]^{* *}$ & $\begin{array}{l}1.04 \\
{[1.02} \\
1.07]^{* *}\end{array}$ \\
\hline & & & & \multirow[t]{5}{*}{$\begin{array}{l}\text { Community mobilisation } \\
\text { at community level (L2) }\end{array}$} & \multirow{5}{*}{$\begin{array}{l}\text { Healthcare } \\
\text { messages at } \\
\text { district level (L1) }\end{array}$} & $\begin{array}{l}\text { Antenatal } \\
\text { care: }\end{array}$ & & \\
\hline & & & & & & Any & & $\begin{array}{l}1.07 \\
{[0.98} \\
1.17]^{*}\end{array}$ \\
\hline & & & & & & $\begin{array}{l}\text { Skilled } \\
\text { birth } \\
\text { attendant }\end{array}$ & & $\begin{array}{l}0.83 \\
{[0.64} \\
1.07]^{*}\end{array}$ \\
\hline & & & & & & $\begin{array}{l}\text { Delivery } \\
\text { at a } \\
\text { health } \\
\text { facility }\end{array}$ & & $\begin{array}{l}1.09 \\
{[1.06} \\
1.13]^{* *}\end{array}$ \\
\hline & & & & & & $\begin{array}{l}\text { ( } N= \\
\text { number } \\
\text { of women } \\
\text { for all } \\
\text { outcomes) }\end{array}$ & & \\
\hline & & & & \multirow{5}{*}{$\begin{array}{l}\text { Community mobilisation } \\
\text { at community level (L2) } \\
\text { involving 'Sure Start' } \\
\text { community field workers } \\
\text { working directly with } \\
\text { ASHAs and } \\
\text { strengthening village } \\
\text { health and sanitation } \\
\text { committees, and health } \\
\text { care messages at district } \\
\text { level (L1) }\end{array}$} & \multirow{5}{*}{$\begin{array}{l}\text { Healthcare } \\
\text { messages at } \\
\text { district level (L1) }\end{array}$} & $\begin{array}{l}\text { Antenatal } \\
\text { care: }\end{array}$ & & \\
\hline & & & & & & Any & & $\begin{array}{l}0.76 \\
{[0.19} \\
3.08]^{*}\end{array}$ \\
\hline & & & & & & $\begin{array}{l}\text { Skilled } \\
\text { birth } \\
\text { attendant }\end{array}$ & & $\begin{array}{l}0.83 \\
{[0.64} \\
1.07]^{*}\end{array}$ \\
\hline & & & & & & $\begin{array}{l}\text { Delivery } \\
\text { at a } \\
\text { health } \\
\text { facility } \\
\text { (any) }\end{array}$ & & $\begin{array}{l}1.05 \\
{[1.02} \\
1.08]^{* *}\end{array}$ \\
\hline & & & & & & $\begin{array}{l}\text { ( } N= \\
\text { number } \\
\text { of women } \\
\text { for all } \\
\text { outcomes) }\end{array}$ & & \\
\hline \multirow{2}{*}{$\begin{array}{l}\text { Baqui [49] } \\
\text { Bangladesh } \\
2003 \text { to }\end{array}$} & \multirow[t]{2}{*}{$\begin{array}{l}\text { Cluster RCT } \\
24 \text { clusters }\end{array}$} & \multirow{2}{*}{$\begin{array}{l}\text { All married women } \\
\text { of reproductive } \\
\text { age }\end{array}$} & \multirow{2}{*}{$\begin{array}{l}\text { 1.Low } \\
\text { 2.Low } \\
\text { 3.High }\end{array}$} & \multirow{2}{*}{$\begin{array}{l}\text { Community mobilisation: } \\
\text { home care with female } \\
\text { mobilisers visiting every }\end{array}$} & \multirow{2}{*}{$\begin{array}{l}\text { Community } \\
\text { mobilisation: } \\
\text { Community care }\end{array}$} & $\begin{array}{l}\text { Antenatal } \\
\text { care: }\end{array}$ & & \\
\hline & & & & & & Any & & 0.76 \\
\hline
\end{tabular}


Table 2 Community participation interventions versus combined health service and community interventions or other types of community-based interventions (Continued)

\begin{tabular}{|c|c|c|c|c|c|c|c|c|}
\hline $\begin{array}{l}\text { Study/Year } \\
\text { published } \\
\text { Country } \\
\text { Duration of } \\
\text { intervention }\end{array}$ & Design & Population & $\begin{array}{l}\text { Risk of } \\
\text { Bias }^{a}\end{array}$ & $\begin{array}{l}\text { Community and Health } \\
\text { Service Intervention }\end{array}$ & $\begin{array}{l}\text { Health Service } \\
\text { Intervention }\end{array}$ & Outcome & OR & $\mathrm{RR}$ \\
\hline \multirow[t]{2}{*}{2006} & & \multirow[t]{2}{*}{ (15-49 years) } & \multirow{2}{*}{$\begin{array}{l}\text { 4.High } \\
\text { 5.High } \\
\text { 6.Low } \\
\text { 7.Low } \\
\text { Overall: } \\
\text { High }\end{array}$} & \multirow[t]{2}{*}{8 months } & \multirow{2}{*}{$\begin{array}{l}\text { with Male } \\
\text { mobilisers visiting } \\
\text { every } 10 \text { months } \\
\text { and Female } \\
\text { mobilisers visiting } \\
\text { every } 4 \text { months }\end{array}$} & & & $\begin{array}{l}{[0.19} \\
3.08]^{*}\end{array}$ \\
\hline & & & & & & $\begin{array}{l}(\mathrm{N}= \\
\text { number } \\
\text { of live } \\
\text { births, \% } \\
\text { = cluster } \\
\text { averages) }\end{array}$ & & $\begin{array}{l}0.94 \\
{[0.89,} \\
1.00]^{* *}\end{array}$ \\
\hline \multirow{3}{*}{$\begin{array}{l}\text { Midhet** } \\
\text { [53] } \\
\text { Pakistan } \\
\text { 1998-2002 }\end{array}$} & \multirow{3}{*}{$\begin{array}{l}\text { Cluster } \\
\text { randomised } \\
\text { trial } \\
32 \text { clusters }\end{array}$} & \multirow{3}{*}{$\begin{array}{l}\text { Women and men of } \\
\text { reproductive age (all } \\
\text { ever-married women } \\
\text { under } 50 \text { years of } \\
\text { age) }\end{array}$} & \multirow{3}{*}{$\begin{array}{l}\text { 1.Low } \\
\text { 2.Unclear } \\
\text { 3.Unclear } \\
\text { 4.Unclear } \\
\text { 5.High } \\
\text { 6.Unclear } \\
\text { 7.Low } \\
\text { Overall: } \\
\text { High }\end{array}$} & \multirow{3}{*}{$\begin{array}{l}\text { Women's and men's } \\
\text { education groups }\end{array}$} & \multirow[t]{3}{*}{$\begin{array}{l}\text { Women's } \\
\text { education groups }\end{array}$} & $\begin{array}{l}\text { Antenatal } \\
\text { care }\end{array}$ & & \\
\hline & & & & & & Any & $1.42[0.99,2.05]^{*}$ & $\begin{array}{l}1.05 \\
{[0.89} \\
1.24]^{*}\end{array}$ \\
\hline & & & & & & $\begin{array}{l}\text { Delivery } \\
\text { at a } \\
\text { health } \\
\text { facility } \\
(\mathrm{N}= \\
\text { number } \\
\text { of } \\
\text { pregnant } \\
\text { women } \\
\text { for all } \\
\text { outcomes) }\end{array}$ & $1.01[0.65,1.56]^{* *}$ & $\begin{array}{l}1.01 \\
{[0.67} \\
1.53]^{* *}\end{array}$ \\
\hline
\end{tabular}

${ }^{\mathrm{a}}$ Risk of Bias tool (Cochrane)

*Adjusted estimate using outcome specific ICC (Page 1)

${ }^{* *}$ Adjusted estimate reported by authors - adjusted for clustering and stratification

***Unadjusted estimate reported by authors

\#Adjusted estimate reported by authors - adjusted for clustering, stratification and baseline covariates

but not the recommended four antenatal visits recommended by the World Health Organisation. Meta-analysis showed no difference in assistance of birth attendants at birth between all community interventions combined, or any community intervention subgroup and control. Women's education group interventions rather than health service or control, and community level mobilisation rather than health care messages at a district level, increased the numbers of women delivering at a health care facility. There was no difference in maternal mortality for meta-analyses between community intervention groups, intervention subgroups and health service, standard care or control. A community-based health promotion intervention [32], including men, women and community members from rural Nepal, showed an improvement in overall knowledge scores for antenatal care and skilled birth attendance.

There were several limitations to the findings of this review. Evidence quality varied across the outcomes for the three main comparisons (refer Additional file 2 GRADE tables). There was moderate to considerable heterogeneity for all outcomes. As the majority of studies were primarily designed to evaluate neonatal rather than maternal outcomes, there were no outcome specific ICC available to calculate adjusted estimates of effect for some maternal outcomes, meaning that some study data for some outcomes, such as institutional delivery, could not be included in the analysis. Studies reported different maternal health knowledge outcome categories which could not be combined in a meta-analysis.

Our review demonstrated improved maternal health outcomes where male community mobilisers were involved in home-based community interventions. Previous evidence suggests that the need to prioritise male involvement in maternal health care education in addition to measures that aim to improve women's education and their status in the family [33]. Involvement of the male family members in maternal health care education is of particular importance in low socioeconomic and uneducated community environments [34]. Intervention strategies involving men and community leaders in maternal health care programs in Bolivia resulted in improved maternal health outcomes in a low resource environment [35]. A study carried out in Maharashtra, India concluded that the maternal mortality ratio was three times higher among women with uneducated husbands compared to 


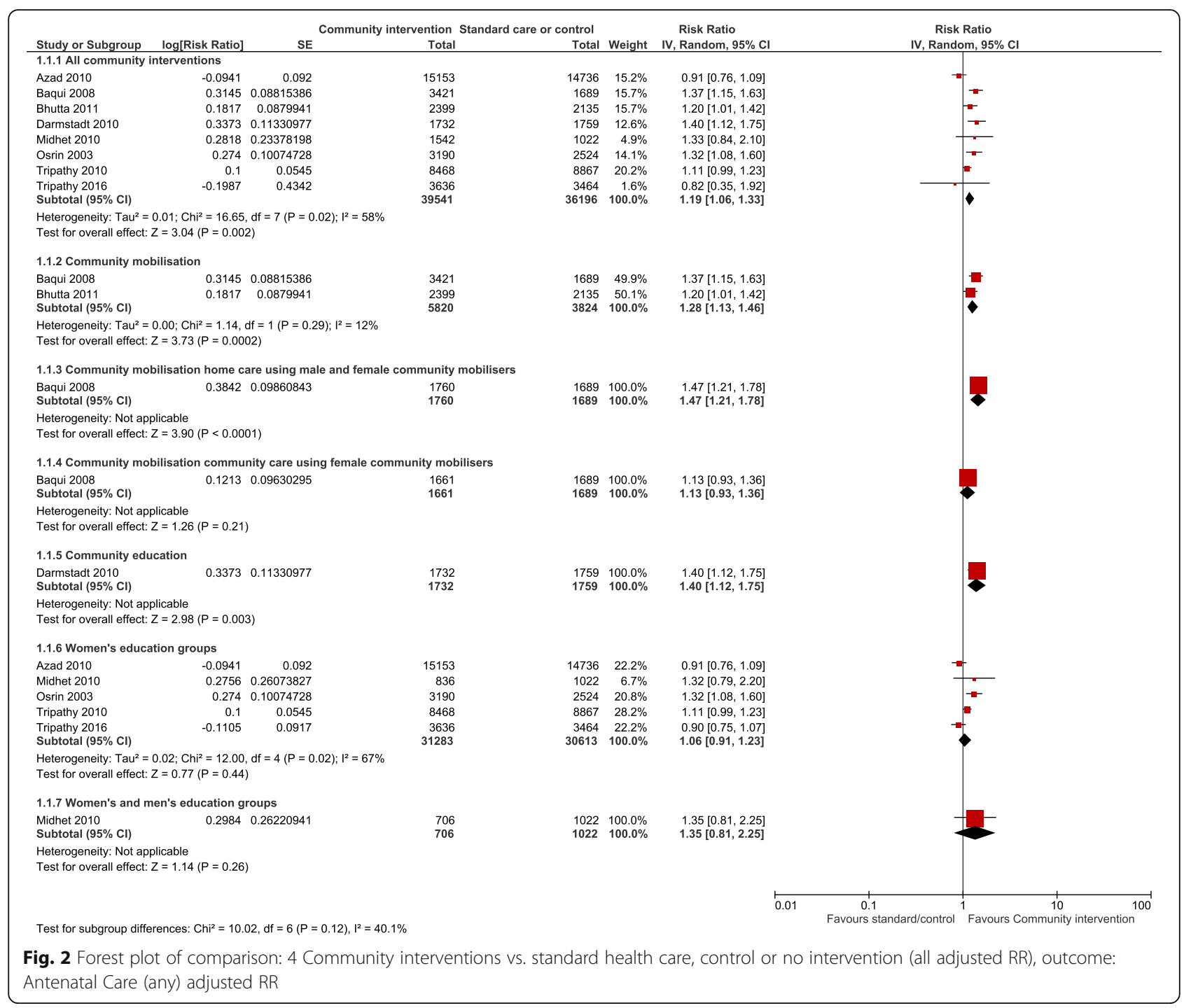

the group of women with college-educated husbands [36]. The involvement of husbands in the utilization of maternal care needs to be included as equally important as the improvement of women's education and their status in the family [33]. Although the involvement of male (fathers-in-law and husbands) members in maternal health care is critical, [37] only one study reported this outcome.

Previous research has concluded that women from deprived communities with poor access to health care and low levels of education have an increased risk of mortality [38]. All community interventions, and use of community mobilisers were more effective than health service, standard care or control, while women's education groups, female mobilisers, women's and men's (couples) education were not. The selection of suitable interventions is critical. This could be one of the reasons that, although worldwide maternal mortality seems to be decreasing, there has not been a similar change for women in rural/remote areas of lower and lower-middle income countries in South Asia [39]. A study carried out in Tamil Nadu, India showed improved maternal health care knowledge among both males and females following education via mobile phone text messaging. Ninety eight percent of participants surveyed responded that text messaging was an effective means of health education [40]. Although this study was carried out in a rural setting, the high level of literacy in Tamil Nadu may have influenced this result. This evidence may therefore not apply to all rural environments of South Asian countries, as use of mobile phone demands many prerequisites such as: ability to read and write, buying a mobile phone, and accessibility to mobile networks in remote locations.

Our meta-analysis found that there was no difference in the risk of maternal death in communities allocated to women's education groups compared to standard care or control. In a systematic review of women's participatory 


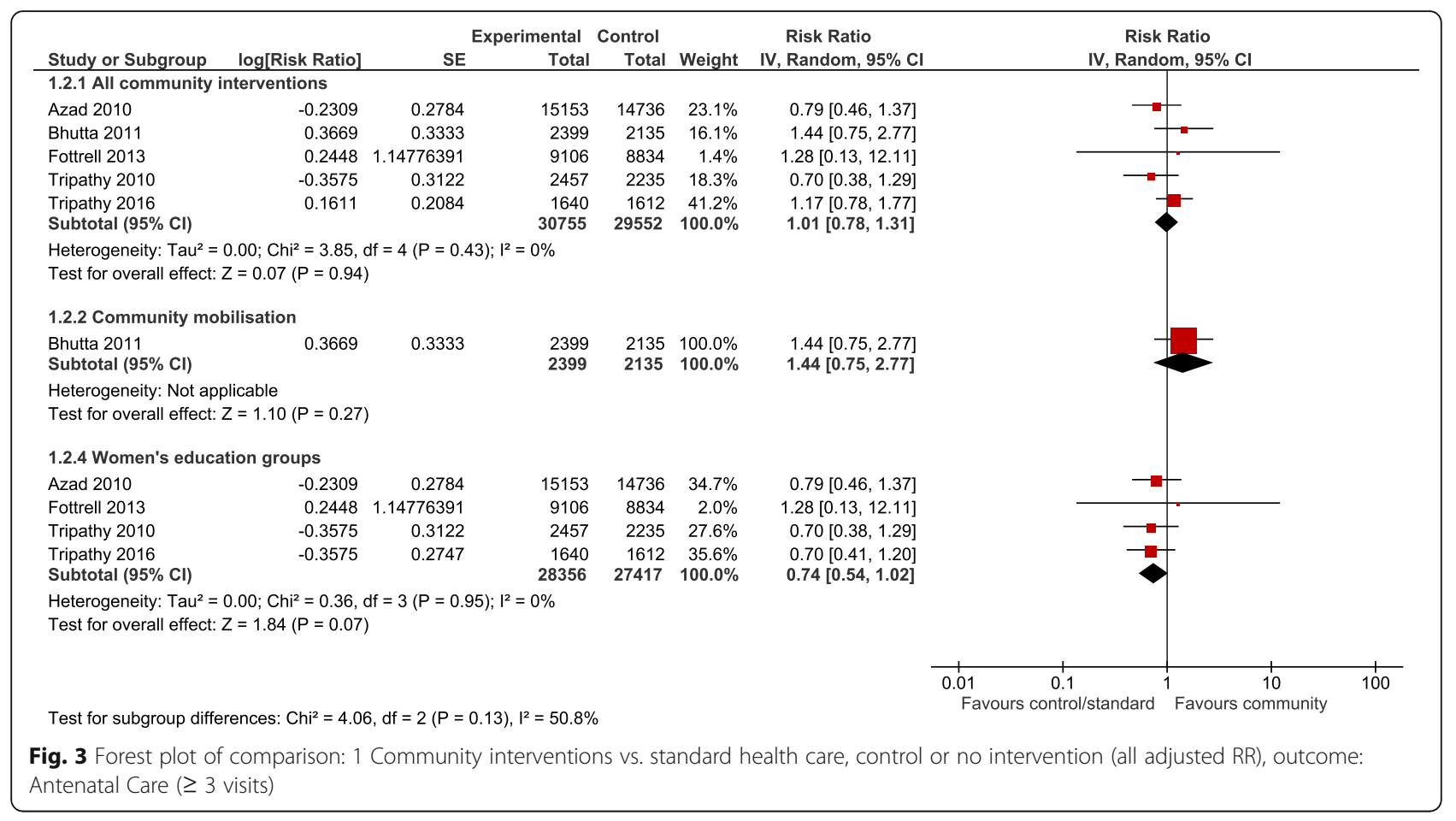

groups in Nepal, Malawi, India and Bangladesh, also no difference in overall maternal mortality was found [41]. After having further divided the women's group according to the percentage of pregnant women attending, Prost [41] found that maternal mortality was halved only in those groups having $>30 \%$ pregnant women (OR 0.51, 95CI 0.29-0.89). Similarly, in our analysis, the only study showing increased risk of mortality, Azad [42], at $3 \%$, had the lowest proportion of pregnant women attending women's education groups out of all the studies in the meta-analysis. It is possible that women's education interventions may need to include a larger proportion of pregnant women in order to be more successful in reducing maternal mortality.

Another systematic review assessing the impact of community interventions on maternal health in resource poor economies, revealed that community-based programs integrated with multiple interventions greatly improved maternal health outcomes [43]. A review of randomised trials aiming to improve antenatal care practice

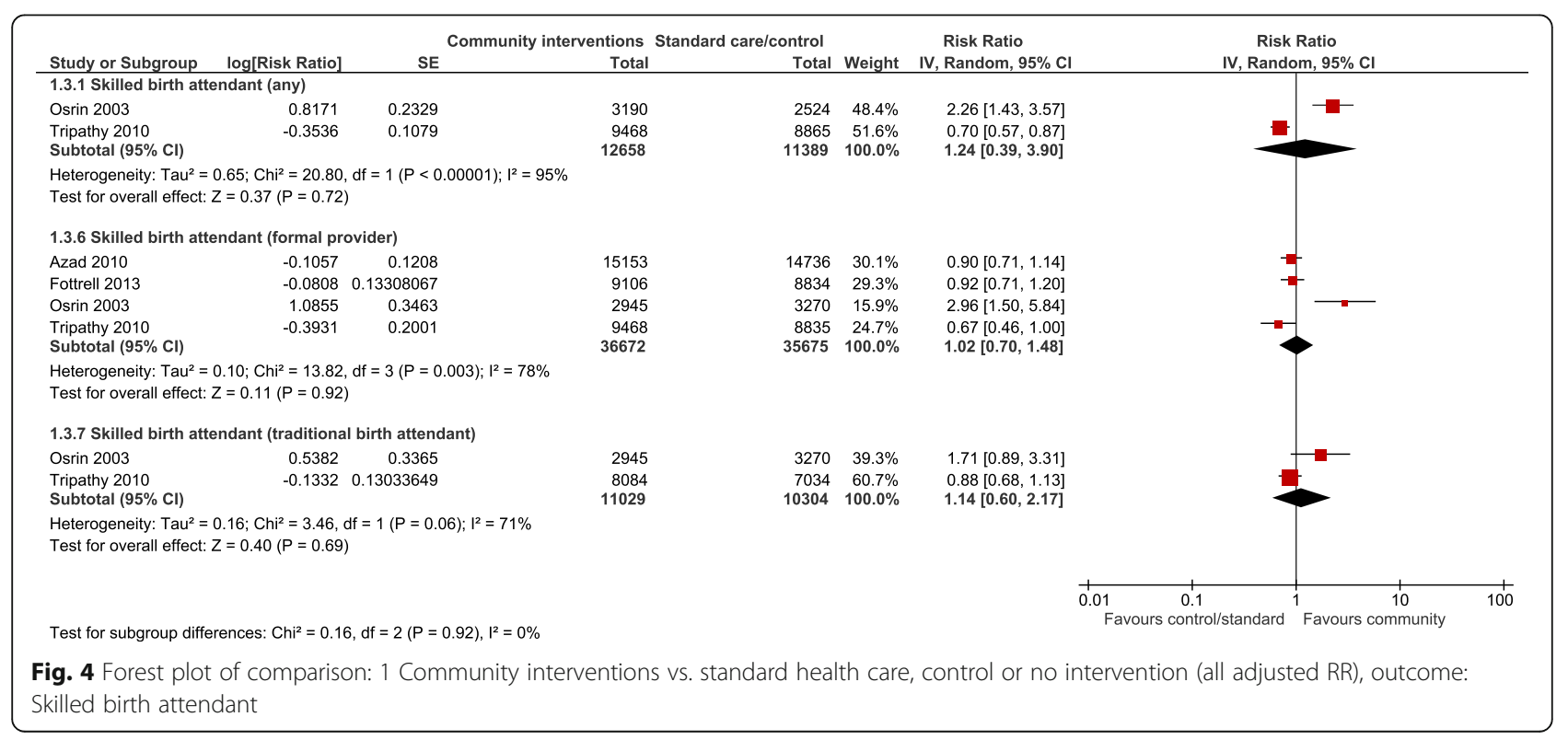




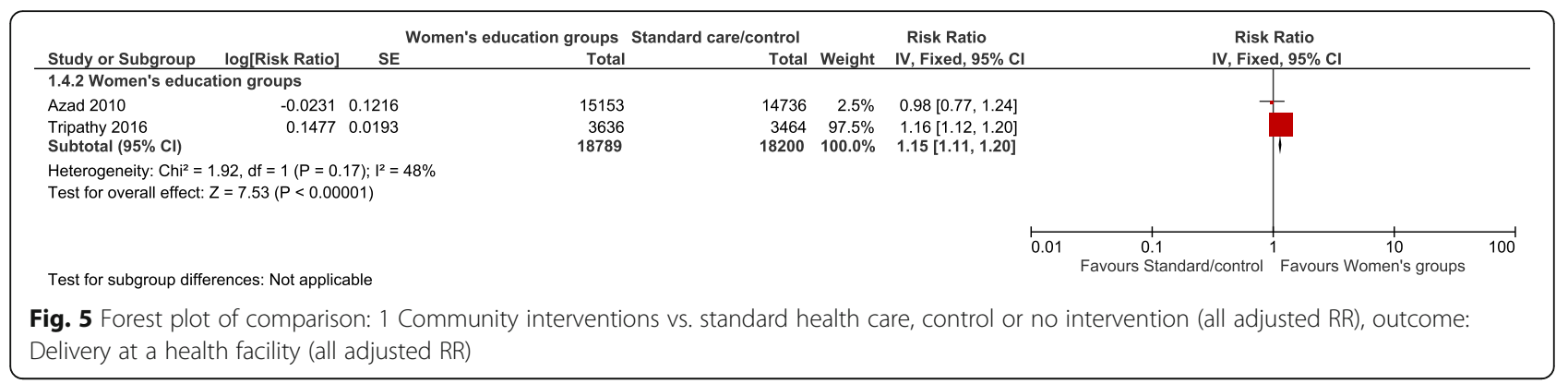

demonstrated a reduction in maternal mortality (OR 0.62 , 95\% CI 0.39-0.98) [44]. The effectiveness of these community level interventions on maternal outcomes challenges the viewpoint that these programs are not worth the cost.

Women's education group interventions did not improve the use of skilled care at birth. It is possible that the involvement of men who control finances and family decisions and improving infrastructure may have improved this outcome. For pregnant women to obtain necessary antenatal visits and skilled care during childbirth, it is necessary to discuss and plan pregnancy care with the men who are responsible for decision-making within the family [45]. Antenatal care is essential to help prevent pregnancy complications and minimize maternal mortality.

Our meta-analysis showed that the numbers of women attending at least one antenatal visit were greater among women receiving any type of community intervention and intervention sub groups. This finding is supported by a study conducted in Nepal that demonstrated improvement in maternal health care outcomes in rural communities using a female facilitator in organizing monthly meetings with women's groups [46].
Increased access to antenatal care, provision of skilled birth attendants and pregnancy care awareness programs at local level contributes to safer pregnancies and childbirth [11]. Previous studies have concluded that delivery in a health care facility offers much needed emotional support to pregnant women [47]. Presence of skilled professionals, lifesaving drugs and equipment help to reduce the risk of complications and death of mother and baby [48]. However, our meta-analysis demonstrated unexpected outcomes of community intervention. For example, none of the interventions were effective in increasing the number of women receiving any level of trained or skilled assistance at birth.

\section{Conclusion}

A range of community interventions are likely to be successful in improving antenatal care attendance. Pregnant women receiving women's education group interventions were more likely to deliver at a health care facility. However, women's education groups were less likely to seek antenatal care or have a formal provider attend at birth. The contributing factors to this are unclear, but it has been previously suggested [41] that the varying

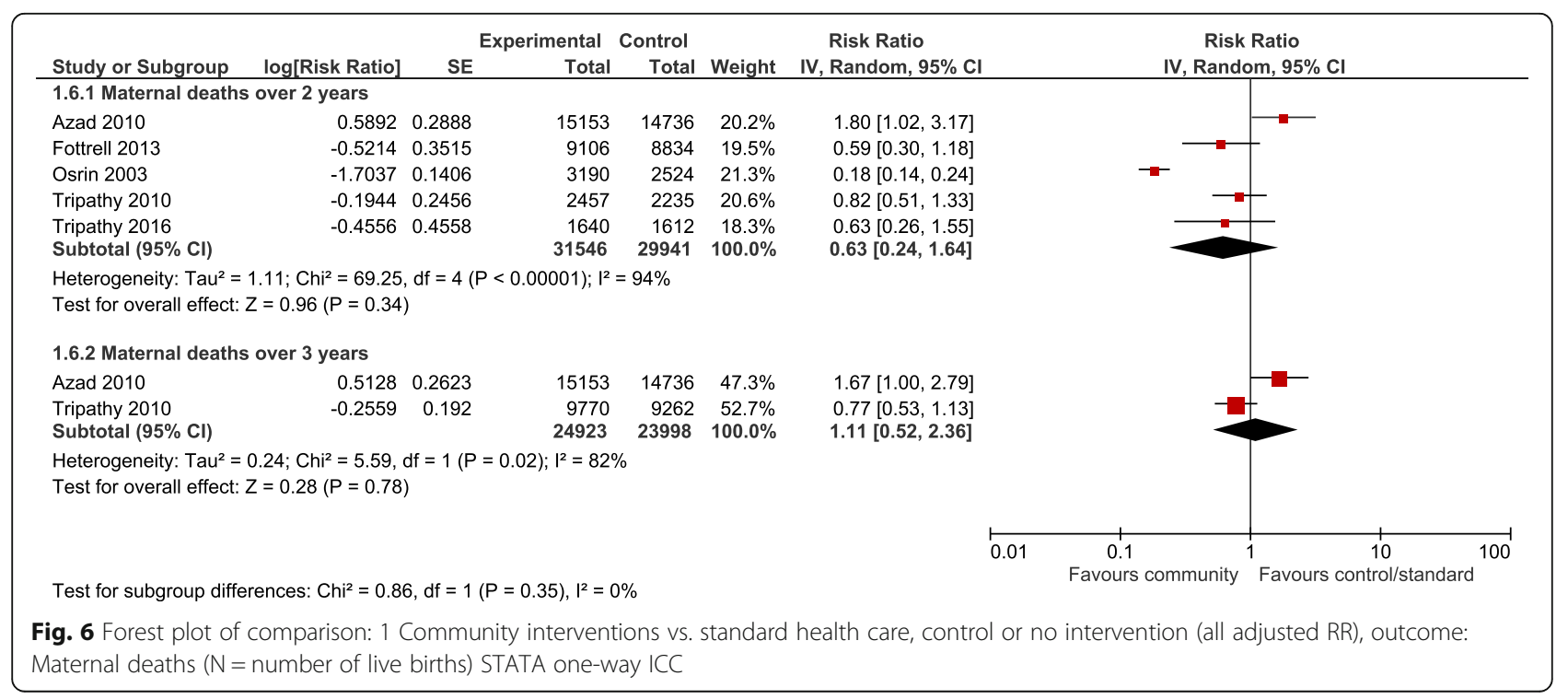


proportion of pregnant women attending women's education interventions among the included studies may have been an important factor. Moderate quality evidence from a single study suggests including male alongside female mobilisers in community mobilisation home care programs [34] may improve the success of women's groups on maternal health care outcomes. Further research is needed on the impact of male involvement in community interventions to supplement women's involvement in community mobilisation, which up until now has been the primary focus, and the impact of couple's education in preference to women only. National health guidelines should include evidence from current systematic reviews of randomised trials, when planning interventions to promote community education on maternal health care.

\section{Additional files}

Additional file 1: The Medline search strategy. (DOCX 19 kb)

Additional file 2: Title of data: GRADE tables. List of outcomes, relative effects $(95 \% \mathrm{Cl})$, number of participants (studies) and certainty of the evidence (GRADE). (PDF $73 \mathrm{~kb}$ )

\section{Abbreviations}

Cl: Confidence interval; GRADE: Grading of Recommendations Assessment, Development and Evaluation.; ICC: Interclass correlation coefficient; MD: Mean difference; OR: Odds ratio; PRISMA: Preferred Reporting in Systematic Reviews and Meta analyses; RCT: Randomised controlled trial; RR: Risk ratio; SD: Standard deviation; SMD: Standardised mean difference; TBA: Traditional Birth Attendant

\section{Acknowledgements}

The authors would like to thank the University of Newcastle, Hunter Medical Research Institute HMRI) and staff and students of the Mothers and Babies Research Centre and Priority Research Centre for Generational Health and Ageing for their support during the study. We thank all the authors who provided additional information. Our thanks also go to HMRI Clinical Research Design and Stats Support Unit (CReDITSS)s for their generous support.

\section{Availability of data and material}

All data generated or analysed during this study are included in this published article [and its Supplementary Information files; search strategy and GRADE tables].

\section{Funding}

BBS received a 50:50 PhD scholarship from the University of Newcastle, Australia.

\section{Authors' contributions}

BBS and $L J$ wrote and registered the protocol. DL and RS commented on the protocol and provided guidance for the searches. BBS, $L$, and DB finalised the search strategies and DB conducted the database searches for related articles. BBS and $L J$ screened the search results, assessed the eligibility of all relevant studies for inclusion and extracted data BBS and $L$ contacted the authors of respective studies for additional information, $\amalg$ conducted the quality assessment and analysed data, both were checked by DB. BBS, LJ, and DB contributed to the final manuscript. RS and DL edited and commented on the draft and provided overall supervision for the systematic review. All authors contributed intellectually to the work. All authors read and approved the final manuscript.
Ethics approval and consent to participate

Not applicable

\section{Consent for publication}

Not applicable

\section{Competing interests}

The authors declare that they have no competing interests.

\section{Publisher's Note}

Springer Nature remains neutral with regard to jurisdictional claims in published maps and institutional affiliations.

\section{Author details}

${ }^{1}$ Mothers and Babies Research Centre, Hunter Medical Research Institute, Lookout Rd, New Lambton Heights, NSW 2305, Australia. ${ }^{2}$ The University of Newcastle, Callaghan, Australia. ${ }^{3}$ Department of Neonatology, John Hunter Hospital, Lookout Rd, New Lambton Heights, NSW 2305, Australia. ${ }^{4}$ Department of Obstetrics and Gynaecology, Sydney Medical School, University of Sydney, Camperdown, Australia. ${ }^{5}$ Priority Research Centre for Generational Health and Ageing, The University of Newcastle, Callaghan, Australia. ${ }^{6}$ Hunter Medical Research Institute, Lot 1 Kookaburra Circuit, New Lambton Heights, NSW 2305, Australia. ${ }^{7}$ Mothers and Babies Research Centre, Hunter Medical Research Institute, Lot 1 Kookaburra Circuit, New Lambton Heights, NSW 2305, Australia. ${ }^{8}$ Department of Endocrinology, John Hunter Hospital, Lookout Rd, New Lambton Heights, NSW 2305, Australia. ${ }^{9}$ Priority Research Centre for Reproductive Science, The University of Newcastle, Callaghan, Australia.

Received: 10 December 2017 Accepted: 3 August 2018 Published online: 10 August 2018

\section{References}

1. Griffin S. Partners in health engage at UNCA: promoting global health through advocacy, education, and fundraising. J Undergrad Neurosci. 2016; IV:1-9.

2. Nour NM. An introduction to maternal mortality. Glastonbury: Rev Obstet Gynecol. 2008;1:77.

3. Pathmanathan I, Liljestrand J. Investing in maternal health: learning from Malaysia and Sri Lanka. Washington, DC: World Bank Publications; 2003.

4. Koblinsky MA. Reducing maternal mortality: learning from Bolivia, China, Egypt, Honduras, Indonesia, Jamaica, and Zimbabwe: World Bank Publications; 2003.

5. Pillai G. Reducing deaths from pregnancy and childbirth. Asia Links. 1993;9: $11-3$

6. Ronsmans C, Graham WJ. Lancet Maternal Survival Series Steering Group. Maternal mortality: who, when, where, and why. Lancet. 2006;368:1189-200.

7. WHO, UNICEF. UNFPA, The World Bank, United Nations Population Division. Trends in maternal mortality: 1990 to 2013. Estimates by WHO, UNICEF. In: UNFPA, The World Bank and the United Nations Population Division. Geneva: World Health Organization; 2014.

8. Graham WJ, Bell JS, Bullough CH. Can skilled attendance at delivery reduce maternal mortality in developing countries. Safe Mother Strat: A Rev Evid. 2001;17:97-130.

9. Paudel DP, Nilgar B, Bhandankar M. Antenatal care service utilization and contributing factors: a community based study in rural Belgaum, Karnataka, India. IOSR J Dent Med Sci. 2013:9:25-31.

10. Simkhada B, Teijlingen ER, Porter M, Simkhada P. Factors affecting the utilization of antenatal care in developing countries: systematic review of the literature. J Adv Nurs. 2008;61:244-60.

11. Baral OP, Vashisth K. Goal, strategies and programme of safe motherhood in Nepal. Acad Voices Multidiscipl J. 2014;3:19-23.

12. Shiffman J. Can poor countries surmount high maternal mortality? Stud Fam Plan. 2000:31:274-89.

13. Brunson J. Confronting maternal mortality, controlling birth in Nepal: the gendered politics of receiving biomedical care at birth. Soc Sci Med. 2010; 71:1719-27.

14. Simkhada B, Porter M, van Teijlingen E. My mother-in-law tells me what to do. Midwives. 2011;14:34-6. 
15. Nwakwuo GC, Oshonwoh FE. Assessment of the level of male involvement in safe motherhood in Southern Nigeria. J Community Health. 2013;38:349-56.

16. Fikree FF, Pasha O. Role of gender in health disparity: the South Asian context. BMJ. 2004;328:823.

17. Stone L. Cultural influences in community participation in health. Soc Sci Med. 1992;35:409-17.

18. Soto EJ, La Vincente S, Clark A, Firth S, Morgan A, Dettrick Z, et al. Investment case for improving maternal and child health: results from four countries. BMC Public Health. 2013;13:601.

19. Bhutta ZA, Gupta I, de'Silva H, Manandhar D, Awasthi S, Hossain SM, et al. Maternal and child health: is South Asia ready for change? BMJ 2004;328: 816-819.

20. Jinadasa WMPK. The study of the potentials of folk-media performance as a form of effective communication in community mobilization. Proc Annual Res Symp. 2006;56

21. Ghosh SK, Patil RR, Tiwari S, Dash AP. A community-based health education programme for bio-environmental control of malaria through folk theatre (Kalajatha) in rural India. Malar J. 2006:5:123.

22. Jinadasa WMPK. Community development programmes and folk-media: a communication model for Sri Lankan rural society. Glob Media J. 2011;2:1-16.

23. Leong CML, Pan SL, Ractham P, Kaewkitipong L. ICT-enabled community empowerment in crisis response: Social media in Thailand flooding 2011. J Assoc Inf Syst. 2015;16:1.

24. Liberati A, Altman DG, Tetzlaff J, Mulrow C, Gøtzsche PC, loannidis JP, et al The PRISMA statement for reporting systematic reviews and meta-analyses of studies that evaluate health care interventions: explanation and elaboration. PLoS Med. 2009;6:e1000100

25. Kululanga LI, Sundby J, Chirwa E. Striving to promote male involvement in maternal health care in rural and urban settings in Malawi-a qualitative study. Reprod Health. 2011;8:36.

26. Leedam E. Traditional birth attendants. Int J Gynecol Obstet. 1985;23:249-74.

27. Covidence systematic review software. http:///covidence.org [Accessed 20 Nov 2017].

28. Schünemann H, Brożek J, Guyatt G, Oxman A. GRADE handbook for grading quality of evidence and strength of recommendations. 2017. The GRADE Working Group, 2013.

29. Review Manager (RevMan). Copenhagen, The Cochrane Collaboration; 2014.

30. Higgins J, Green S, editors. Cochrane handbook for systematic reviews of interventions Chichester. West Sussex: Wlley-Blackwell; 2011

31. Pagel C, Prost A, Lewycka S, Das S, Colbourn T, Mahapatra R, et al. Intracluster correlation coefficients and coefficients of variation for perinatal outcomes from five cluster-randomised controlled trials in low and middleincome countries: results and methodological. Trials. 2011;12:151.

32. Sharma BB, Loxton DJ, Murray H, Angeli GL, Oldmeadow C, Chiu S, Smith R. Singing Health Messages is Effective in Transmitting Knowledge Regarding Antenatal Care in Nepal. Am J Obstet Gynecol. Under review.

33. Halim N, Bohara AK, Ruan X. Healthy mothers, healthy children: does maternal demand for antenatal care matter for child health in Nepal? Health Policy Plan. 2011;26:242-56.

34. Bhalerao VR, Galwankar M, Kowli SS, Kumar R, Chaturvedi RM. Contribution of the education of the prospective fathers to the success of maternal health care programme. J Postgrad Med. 1984;30:10-2.

35. Morrison J, Tamang S, Mesko N, Osrin D, Shrestha B, Manandhar M, et al. Women's health groups to improve perinatal care in rural Nepal. BMC Pregnancy Childbirth. 2005;5(6):1-12.

36. Ganatra BR, Coyaji KJ, Rao VN. Too far, too little, too late: a communitybased case-control study of maternal mortality in rural west Maharashtra, India. Bull World Health Organ. 1998;76:591-8.

37. Chattopadhyay A. Men in maternal care: evidence from India. J Biosoc Sci. 2012:44:129-53.

38. Bhandari A, Gordon M, Shakya G. Reducing maternal mortality in Nepal. BJOG. 2011:118:26-30

39. Dhakal S. Maternal mortality falls in Nepal but inequalities exist. Lancet. 2007:370:1301

40. Datta SS, Ranganathan P, Sivakumar KS. A study to assess the feasibility of text messaging service in delivering maternal and child healthcare messages in a rural area of Tamil nadu, India. Austral Med J. 2014;7:175-80

41. Prost A, Colbourn T, Seward N, Azad K, Coomarasamy A, Copas A, et al. Women's groups practising participatory learning and action to improve maternal and newborn health in low-resource settings: a systematic review and meta-analysis. Lancet. 2013:381:1736-46.

42. Azad K, Barnett S, Banerjee B, Shaha S, Khan K, Rego AR, et al. Effect of scaling up women's groups on birth outcomes in three rural districts in Bangladesh: a cluster-randomised controlled trial. Lancet. 2010;375:1193-202.

43. Nyamtema AS, Urassa DP, van Roosmalen J. Maternal health interventions in resource limited countries: a systematic review of packages, impacts and factors for change. BMC Pregnancy Childbirth. 2011;11:30.

44. Kidney E, Winter HR, Khan KS, Gülmezoglu AM, Meads CA, Deeks JJ, et al. Systematic review of effect of community-level interventions to reduce maternal mortality. BMC Pregnancy Childbirth. 2009;9:2.

45. Li J. Gender inequality, family planning, and maternal and child care in a rural Chinese county. Soc Sci Med. 2004;59:695-708.

46. Manandhar DS, Osrin D, Shrestha BP, Mesko N, Morrison J, Tumbahangphe $\mathrm{KM}$, et al. Effect of a participatory intervention with women's groups on birth outcomes in Nepal: cluster-randomised controlled trial. Lancet. 2004; 364:970-9.

47. Tumblin A, Simkin P. Pregnant women's perceptions of their nurse's role during labor and delivery. Birth. 2001;28:52-6.

48. Kesterton AJ, Cleland J, Sloggett A, Ronsmans C. Institutional delivery in rural India: the relative importance of accessibility and economic status. BMC Pregnancy Childbirth. 2010;10:30.

49. Baqui AH, Williams E, El-Arifeen S, Applegate JA, Mannan I, Begum N, et al. Effect of community-based newborn care on cause-specific neonatal mortality in Sylhet district, Bangladesh: findings of a cluster-randomized controlled trial. J Perinatol. 2016;36:71-6.

50. Bhutta ZA, Soofi S, Cousens S, Mohammad S, Memon ZA, Ali I, et al. Improvement of perinatal and newborn care in rural Pakistan through community-based strategies: a cluster-randomised effectiveness trial. Lancet. 2011;377:403-12.

51. Darmstadt GL, Choi Y, Arifeen SE, Bari S, Rahman SM, Mannan I, et al. Evaluation of a cluster-randomized controlled trial of a package of community-based maternal and newborn interventions in Mirzapur, Bangladesh. PLoS One. 2010:5:e9696.

52. Fottrell E, Azad K, Kuddus A, Younes L, Shaha S, Nahar T, Aumon BH, Hossen M, Beard J, Hossain T, et al. The effect of increased coverage of participatory women's groups on neonatal mortality in Bangladesh: A cluster randomized trial. JAMA Pediatr. 2013;167:816-25.

53. Midhet F, Becker S. Impact of community-based interventions on maternal and neonatal health indicators: results from a community randomized trial in rural Balochistan, Pakistan. Reprod Health. 2010;7:10.

54. Osrin D, Mesko N, Shrestha BP, Shrestha D, Tamang S, Thapa S, Tumbahangphe KM, Shrestha JR, Manandhar MK, Manandhar DS, et al. Implementing a community-based participatory intervention to improve essential newborn care in rural Nepal. Trans R Soc Trop Med Hyg. 2003; 97:18-21

55. Tripathy P, Nair N, Barnett S, Mahapatra R, Borghi J, Rath S, et al. Effect of a participatory intervention with women's groups on birth outcomes and maternal depression in Jharkhand and Orissa, India: a cluster-randomised controlled trial. Lancet. 2010;375:1182-92.

56. Tripathy P, Nair N, Sinha R, Rath S, Gope RK, Rath S, Roy SS, Bajpai A, Singh $V$, Nath $V$. Effect of participatory women's groups facilitated by Accredited Social Health Activists on birth outcomes in rural eastern India: a clusterrandomised controlled trial. Lancet Glob Health. 2016:4:e119-e28.

57. Acharya A, Lalwani T, Dutta R, Knoll Rajaratnam J, Ruducha J, Varkey LC, et al. Evaluating a large-scale community-based intervention to improve pregnancy and newborn health among the rural poor in India. Am J Public Health. 2015:105:144-52. 